\title{
Curcumin Provides Incomplete Protection of the Kidney in Ischemia Reperfusion Injury
}

\author{
F. T. HAMMAD ${ }^{1}$, S. AL-SALAM ${ }^{2}$, L. LUBBAD ${ }^{1}$ \\ ${ }^{1}$ Department of Surgery and ${ }^{2}$ Department of Pathology, Faculty of Medicine and Health Sciences, \\ United Arab Emirates University, Al Ain, United Arab Emirates
}

Received April 16, 2012

Accepted June 8, 2012

On-line August 8, 2012

\begin{abstract}
Summary
Curcumin, a component of the spice turmeric, was shown to have a protective effect on acute kidney injury markers following ischemia-reperfusion injury (IRI). However, its effect on glomerular and tubular renal functions following IRI is not known and this data is probably of more clinical relevance. In this study, curcumin was tested for its effect on renal functional parameters following two different periods of warm IRI in the rat. Groups V-30 $(n=10)$ and C-30 $(n=10)$ underwent ischemia for 30 minutes whereas groups V-45 $(n=8)$ and C-45 $(n=8)$ underwent ischemia for 45 minutes. $C-30$ and $C-45$ received oral curcumin $(200 \mathrm{mg} / \mathrm{kg} /$ day) whereas $\mathrm{V}-30$ and V-45 received a vehicle. The left renal artery blood flow was measured by a flowmeter before and 15 minutes after reperfusion. Serum TNF-a was measured before and 2 days after ischemia. The function of both kidneys was measured 2 days following ischemia using clearance technique. IRI caused significant increase in TNF-a in all groups. Curcumin significantly ameliorated the ischemiainduced alterations in serum TNF-a and associated histological changes but did not affect the alterations in renal artery blood flow, glomerular (glomerular filtration rate, renal blood flow) or tubular (urinary volume, urinary sodium and fractional excretion of sodium) functions following 30 or $45 \mathrm{~min}$ of IRI.
\end{abstract}

\section{Key words}

Ischemia-reperfusion • Curcumin • Renal function

\section{Corresponding author}

Fayez T. Hammad, Department of Surgery, Faculty of Medicine and Health Sciences, PO Box 17666, Al Ain, United Arab Emirates. Fax: 009713 7672067. E-mail: fayez@mail2doctor.com

\section{Introduction}

Renal ischemia-reperfusion injury (IRI) is an invariable consequence of several conditions including renal transplantation (Weight et al. 1996). It has been well established that renal IRI is associated with alterations in renal functions (Axelsen and Cartwright 1979, Brady et al. 1996). These alterations in renal functions are induced by both the ischemic insult and the release of oxygen-derived free radicals associated with the reperfusion process (McCord 1985, Reilly et al. 1991).

Curcumin (diferuloylmethane) is a phenolic compound which forms the active ingredient of the dietary spice turmeric (Curcuma longa). Similar to other herbal therapies, curcumin is being used relatively frequently worldwide due to people's preference to use herbal agents over conventional medications especially in certain cultures. Curcumin has been shown to exhibit protective effect in several conditions (Nagabhushan and Bhide 1992, Jordan and Drew 1996, Mahady et al. 2002, Salh et al. 2003, LoTempio et al. 2005) due to its antioxidant (Sharma 1976, Sugiyama et al. 1996), antiinflammatory (Srimal and Dhawan 1973), antimicrobial (Jordan and Drew 1996, Mahady et al. 2002) and anticarcinogenic activities (Nagabhushan and Bhide 1992, LoTempio et al. 2005).

In renal IRI, limited number of studies has demonstrated a protective effect of curcumin on the kidney (Shoskes 1998, Shahed et al. 2001, Bayrak et al. 2008). For example, 24 hours following 45 minutes of bilateral warm renal ischemia, Bayrak and colleagues have shown that curcumin significantly ameliorated the 
alterations in the serum level of cystatin $\mathrm{C}$ and glutathione peroxidase and the serum and tissue levels of malondialdehyde and nitric oxide (Bayrak et al. 2008). However, the attenuation in these markers does not necessarily indicate an improvement in the glomerular and tubular renal functions and the effect of curcumin on the glomerular and tubular renal function was not investigated previously. Thus, the aim of this study was to investigate the effect of curcumin on the alterations in renal functional parameters following different periods of warm IRI in the rat.

\section{Methods}

Studies were performed in male Wistar rats weighing 280-321g at the time of IRI. Rats were housed in standard cages and kept in a 12-hour light-dark cycle at $20{ }^{\circ} \mathrm{C}$. They were fed a standard rat chow and had free access to water. Animals were fasted for 12 hours before the experimental procedures but had water ad libitum. The experimental protocol was approved by the local animal research ethics committee.

\section{Ischemic injury and peri-operative renal artery blood} flow measurements

The following procedures were carried out under aseptic conditions. Animals were anesthetized with ketamine hydrochloride (70 $\mathrm{mg} / \mathrm{kg}$, intraperitoneally, Pantex Holland B.V., Holland) and Pentobarbital Sodium (20 $\mathrm{mg} / \mathrm{kg}$, intraperitoneally, Sigma Life Science, St Louis, USA). With minimal dissection, the left renal artery was exposed via a flank incision and was occluded using microvascular non-traumatic bulldog clamp. Following a warm ischemia of 30 or $45 \mathrm{~min}$ depending on the group, the microvascular clamp was removed to allow reperfusion.

The total left renal artery blood flow (ml/minute) was measured by careful placement of a 0.7PSB probe (TS420 Perivascular Flowmeter, Transonic Systems Inc. Netherlands) on the distal part of the artery. The pre-ischemic measurement (LRABFPre) was obtained 10 minutes after the isolation of the renal vessels and the post-ischemic measurement (LRABF-Post) was obtained 15 minutes after release of the bulldog clamp. On both occasions, measurements were obtained for 5 minutes and the mean left renal artery blood flow was calculated. At the end, the wound was closed in layers.

\section{Curcumin/Vehicle administration}

Curcumin (Sigma Life Science, St Louis, USA) was dissolved in $1 \mathrm{ml}$ of the vehicle $(0.5 \%$ carboxymethylcellulose, Sigma Life Science, St Louis, USA) and administered by gavage immediately after preparation as single daily dose of $200 \mathrm{mg} / \mathrm{kg}$. Control animal received only $1 \mathrm{ml}$ of the vehicle. In all groups, treatment was commenced five days prior to the ischemia and an extra-dose was given one day post-ischemia.

\section{Experimental groups}

Animals were divided into 4 groups:

1. V-30 group $(n=10)$ : Rats which underwent renal ischemia for $30 \mathrm{~min}$ and received vehicle only.

2. C-30 group $(n=10)$ : Rats which underwent renal ischemia for $30 \mathrm{~min}$ and received curcumin dissolved in the vehicle.

3. $\mathrm{V}-45$ group $(\mathrm{n}=8)$ : Rats which underwent renal ischemia for $45 \mathrm{~min}$ and received vehicle only.

4. C-45 group $(n=8)$ : Rats which underwent renal ischemia for $45 \mathrm{~min}$ and received curcumin dissolved in the vehicle.

\section{Surgical procedure in the terminal experiment}

All rats underwent terminal experiment two days following IRI. Animals were anaesthetised with pentobarbital sodium $(60-70 \mathrm{mg} / \mathrm{kg}$, intraperitoneally; Sigma Life Science, St Louis, USA) and the trachea was cannulated. Following cannulation of a femoral vein with polyethylene tubing (PE-50), anaesthesia was maintained by a continuous infusion of pentobarbital sodium $(12.5 \mathrm{mg} / \mathrm{kg} / \mathrm{hr})$ and a sustaining infusion of $0.9 \%$ saline was established at a rate of $50 \mu \mathrm{l} / \mathrm{min}$ using an infusion pump. A femoral artery was cannulated with similar tubing used in the femoral vein and the tip of the cannula was positioned just below the level of the left renal artery. The cannula was connected to a pressure transducer (Memscap, Skoppum, Norway). The blood pressure signal was amplified using a bridge Amp (ADInstruments, Castle Hill, Australia), digitised using Power Lab 4/30 and Lab Chart version 6 software (ADInstruments, Australia) and displayed on a computer screen. The arterial cannula was also used to obtain blood samples throughout the procedure as required. Both kidneys were exposed through a midline abdominal incision and the upper ureters were cannulated with polyethylene tubing (PE-10) for the collection of urine into pre-weighed micro-capped tubes. The urine volume was determined gravimetrically. 
On completion of surgery, the sustaining infusion of $0.9 \%$ saline was replaced by one composed of fluorescein isothiocyanate-inulin (FITC-inulin, SigmaAldrich, St Louis, USA) $(2.5 \mathrm{mg} / \mathrm{ml})$ and paraaminohippuric acid (PAH, Sigma-Aldrich, St Louis, USA) $(0.2 \% \mathrm{w} / \mathrm{v})$ in $0.9 \%$ saline. A priming dose of $2 \mathrm{ml}$ of the same solution was infused over 2 minutes. Animals were allowed 2 hours to equilibrate before being subjected to the experimental protocol.

\section{Experimental protocol and assays}

The experimental protocol consisted of two 30minute clearance periods. Arterial blood samples $(0.4 \mathrm{ml})$ taken at the beginning and end of the clearance periods were immediately centrifuged. Plasma samples (125 $\mu \mathrm{l})$ were frozen to be assayed later. The plasma was replaced by an equal volume of saline and the erythrocytes were resuspended by gentle vortexing and returned to the animal. The haematocrit was determined. Finally, after euthanizing the animals, the kidneys were weighed and stored in $10 \%$ neutral formalin for histological examination.

Urine and plasma samples were assayed for sodium level using a flame photometer (Corning, Halstead, Essex, England). Inulin and PAH contents were determined using modified techniques described by Gekle and Silbernagl (1993) and Bratton and Marshall (1939), respectively. Glomerular filtration rate (GFR) was estimated from the clearance of inulin. Renal blood flow (RBF) was calculated using the formula $[\mathrm{RBF}=\mathrm{ERPF} /(1$-hematocrit $)]$, where the PAH clearance was used to estimate ERPF (effective renal plasma flow). The values of GFR, RBF, urine volume (UV), urinary sodium $\left(\mathrm{U}_{\mathrm{Na}} \mathrm{V}\right)$ and fractional excretion of sodium $\left(\mathrm{FE}_{\mathrm{Na}}\right)$ were calculated as the average of the two clearance periods and were corrected for kidney weight.

The serum level of TNF- $\alpha$ (tumor necrosis factor- $\alpha$ ) was measured just before ischemia and one hour after reperfusion using Quantikine TNF- $\alpha$ Rat ELISA kit (R\&D Systems, Minneapolis, USA) according to the manufacturer's instructions. The samples were tested in duplicate. Results were expressed as picograms of TNF- $\alpha$ per milliliter serum (pg/ml).

\section{Histological studies}

Kidneys were embedded in paraffin and five $\mu \mathrm{m}$ sections were stained with haematoxylin and eosin. Histological examination and scoring were performed blindly by a pathologist. A numerical score was used to define the degree of tubular cell necrosis as described previously (Mohan et al. 2006) (0: no necrosis; 1: few focal necrotic spots; 2 : tubular necrosis in approximately half of the field; 3 , tubular necrosis in approximately two thirds of the field; 4: approximately the entire field was necrotic).

\section{Statistical analysis}

Statistical analysis was performed using SPSS V16.0. Results were expressed as means \pm SEM. Oneway factorial ANOVA was used for comparison of variables between V-30 and C-30, V-45 and C-45 and between the control and ischemic kidneys within each group. TNF- $\alpha$ levels and left renal artery blood flow in each group before and after ischemia, were compared using the Student's paired $t$-test. P value of less than 0.05 was considered statistically significant.

\section{Results}

The basal mean arterial blood pressure and heart rate in the V-30 and C-30 groups were similar (118 \pm 5 vs. $124 \pm 4$ and $452 \pm 10$ vs. $449 \pm 6$, respectively). Similarly, there was no difference in these variables between V-45 and C-45 (123 \pm 4 vs. $121 \pm 4$ and $443 \pm 11$ vs. $455 \pm 10$, respectively) ( $\mathrm{P}>0.05$ for all these comparisons).

\section{Left renal artery blood flow (flowmeter measurements)}

As shown in Figure 1, in both V-30 and C-30 groups, LRABF-Post was significantly lower than LRABF-Pre $(2.4 \pm 0.4$ vs. $3.4 \pm 0.3, \mathrm{P}=0.03$ and $2.7 \pm 0.3$ vs. $3.6 \pm 0.2, \mathrm{P}=0.02$, respectively).

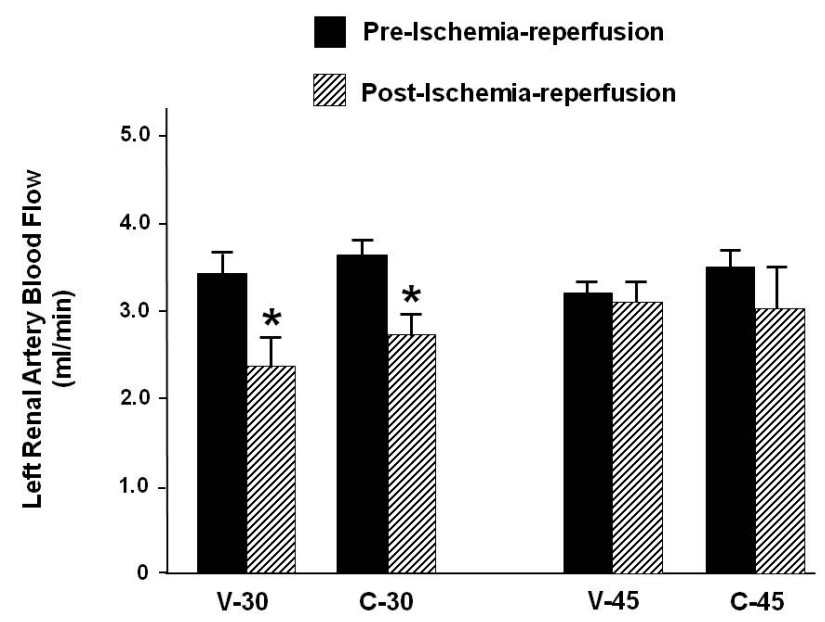

Fig. 1. The pre- and post-ischemia total left renal blood flow in both the vehicle (V-30 and V-45) and curcumin (C-30 and C-45) groups following 30 and 45 minutes of renal ischemia. Values represent mean \pm SEM. $*$ indicates statistical significance when compared to the pre-ischemia levels. 

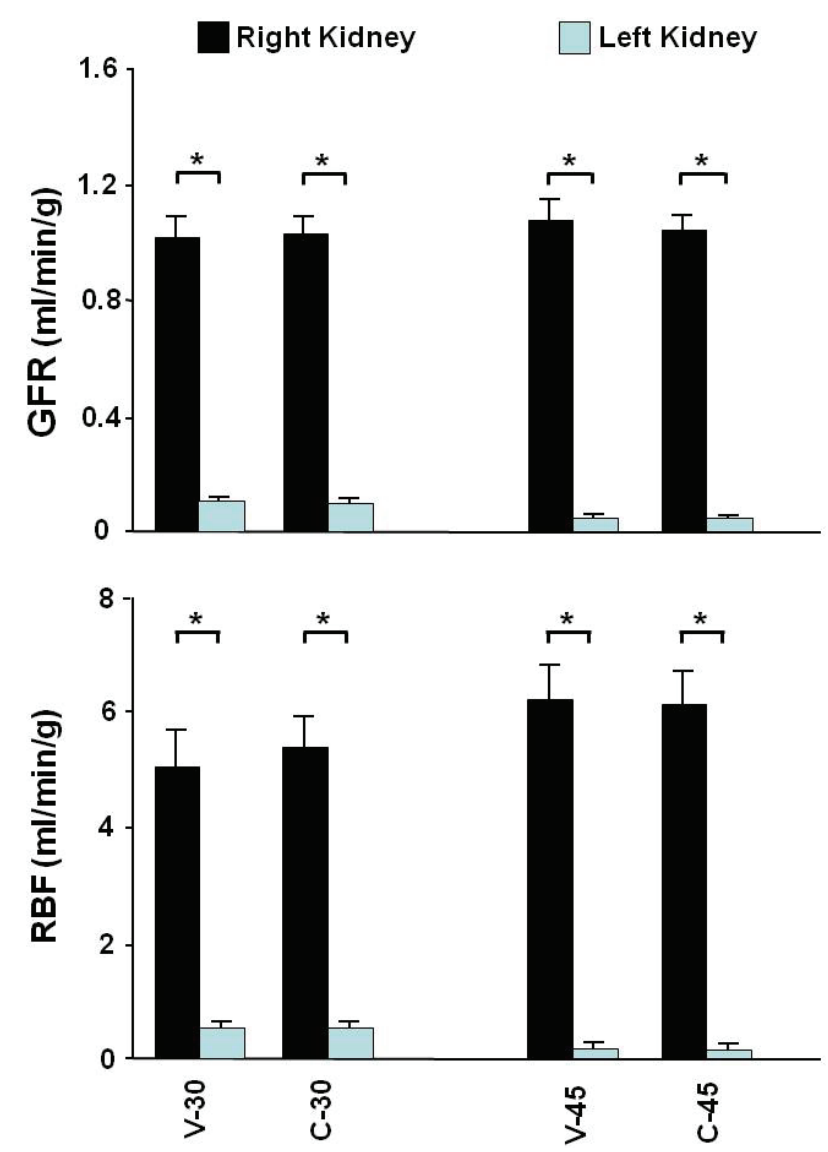

Fig. 2. The glomerular filtration rate (GFR) and renal blood flow (RBF) in the vehicle (V-30 and V-45) and curcumin (C-30 and C-45) groups following 30 and 45 minutes of renal ischemia. Values are represent mean \pm SEM. * indicates statistical significance when compared to the right non-ischemic kidney.

In V-45 and C-45 groups, the LRABF-Post was lower than LRABF-Pre but did not reach statistical significance $(3.1 \pm 0.3$ vs. $3.2 \pm 0.1, \mathrm{P}=0.48$ and $3.0 \pm 0.5$ vs. $3.5 \pm 0.2, \mathrm{P}=0.39$, respectively) (Figure 1).

There was no difference of the LRABF-Post between $\mathrm{C}-30$ and $\mathrm{V}-30$ or between $\mathrm{C}-45$ and V-45 groups $(\mathrm{P}>0.05$ for all).

Glomerular and tubular function of right and left kidneys 2 days after ischemia

In the V-30 group, left renal GFR two days following the IRI was $11 \%$ that of the right non-ischemic kidney $(0.11 \pm 0.02$ vs. $0.99 \pm 0.09, \mathrm{P}<0.0001)$ (Figure 2). Similarly, left $\mathrm{RBF}$ was $11 \%$ of the right $\mathrm{RBF}$ $(0.54 \pm 0.13$ vs. $5.01 \pm 0.71, \mathrm{P}<0.0001)$. The $\mathrm{UV}$ and $\mathrm{U}_{\mathrm{Na}} \mathrm{V}$ in the two kidneys were comparable $(10.9 \pm 3.1$ vs. $14.5 \pm 4.7, \mathrm{P}=0.52$ and $1.40 \pm 0.41$ vs. $2.61 \pm 1.15, \mathrm{P}=0.34$, respectively) (Figure 3). However, left renal $\mathrm{FE}_{\mathrm{Na}}$ was significantly higher than the one in the right kidney $(12.3 \pm 4.0$ vs. $2.1 \pm 0.9, \mathrm{P}=0.02)$.

In the C-30 group, left renal GFR and RBF were
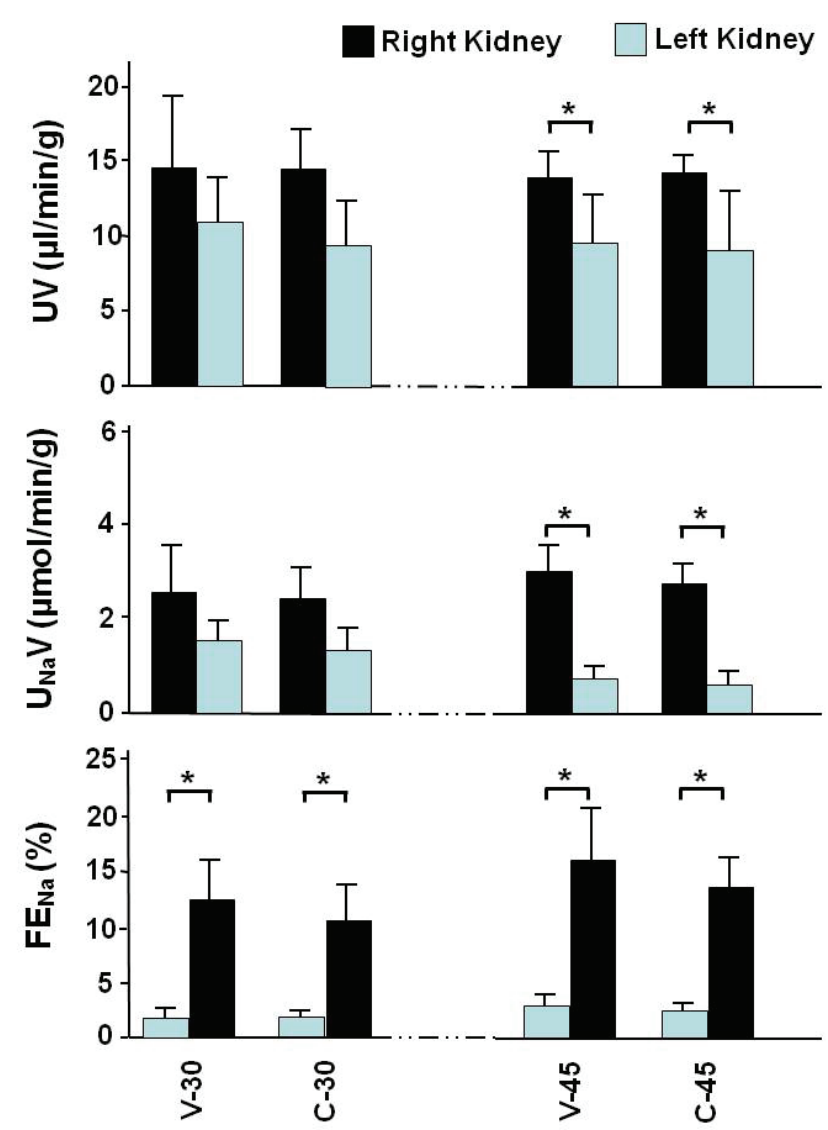

Fig. 3. The tubular functional parameters including urine volume (UV), urinary sodium $\left(\mathrm{U}_{\mathrm{Na}} \mathrm{V}\right)$ and fractional excretion of sodium $\left(\mathrm{FE}_{\mathrm{Na}}\right)$ in the vehicle (V-30 and $\left.\mathrm{V}-45\right)$ and curcumin (C-30 and $\mathrm{C}-45)$ groups. Values represent mean \pm SEM. * indicates statistical significance when compared to the right non-ischemic kidney.

significantly lower than the right kidney (9\% and $10 \%$, respectively ( $\mathrm{P}<0.0001$ for both comparisons) (Figure 2). Similar to V-30, there was no significant difference in $\mathrm{UV}$ and $\mathrm{U}_{\mathrm{Na}} \mathrm{V}$ between the two kidneys $(\mathrm{P}>0.05$ for both variables). However, left renal $\mathrm{FE}_{\mathrm{Na}}$ was significantly higher than the right kidney $(9.7 \pm 3.0$ vs. $2.0 \pm 0.4(\mathrm{P}=0.02)$ (Figure 3).

In the V-45 group, GFR of the left ischemic kidney was $4 \%$ that of the right kidney $(0.04 \pm 0.02$ vs. $1.08 \pm 0.08, \mathrm{P}<0.0001$ ) (Figure 2). Similarly, left RBF was $3 \%$ of right $\mathrm{RBF}(0.22 \pm 0.05$ vs. $6.28 \pm 0.68, \mathrm{P}<0.0001)$. As shown in Figure 3, all left renal tubular parameters were significantly different from those in the right kidney (UV: $9.3 \pm 3.2$ vs. $13.9 \pm 1.6(\mathrm{P}=0.04) ; \mathrm{U}_{\mathrm{Na}} \mathrm{V}: 0.66 \pm 0.27$ vs. $3.00 \pm 0.53 \quad(\mathrm{P}=0.001) ; \quad \mathrm{FE}_{\mathrm{Na}}: \quad 16.0 \pm 4.8 \quad$ vs. $2.8 \pm 0.7$ $(\mathrm{P}=0.03)$.

In the C-45 group, both left renal GFR and RBF were $3 \%$ of the value in the right kidney $(\mathrm{P}<0.0001$ for both variables) (Figure 2). As shown in Figure 3, all tubular parameters of the left kidney were significantly different from those in the right kidney $(\mathrm{P}<0.05$ for all 
variables).

Administration of curcumin in the C-30 and C-45 groups did not affect any of the glomerular or tubular functions of the left kidney in both groups in comparison to $\mathrm{V}-30$ and $\mathrm{V}-45$ groups, respectively ( $\mathrm{P}>0.05$ for all variables). In addition, there was no difference between these variables among the contralateral non-ischemic kidneys in the 30 minute (V-30 and C-30) or 45 minute (V-45 and C-45) ischemia groups ( $\mathrm{P}>0.05$ for all variables and in all groups).

\section{Serum level of $T N F-\alpha$}

In $\mathrm{V}-30$ and $\mathrm{C}-30$ groups, TNF- $\alpha$ one hour following ischemia was significantly higher than the preischemia value $(5.6 \pm 0.9$ vs. $2.4 \pm 0.2(\mathrm{P}=0.02)$ and $3.6 \pm 0.6$ vs. $2.2 \pm 0.2(\mathrm{P}=0.04)$, respectively) (Figure 4$)$.

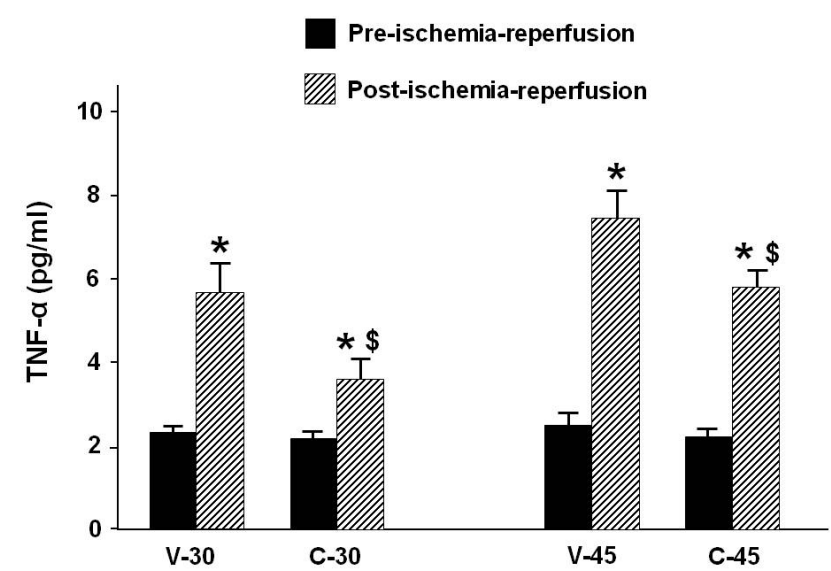

Fig. 4. The pre- and post-ischemia serum levels of TNF-a in both the vehicle (V-30 and V-45) and curcumin (C-30 and C-45) groups following 30 and 45 minutes of renal ischemia. Values represent mean \pm SEM. * indicates statistical significance when compared to the pre-ischemia levels, $\$$ indicates statistical significance when compared to the $\mathrm{V}$ groups.

In V-45 and C-45 groups, TNF- $\alpha$ after ischemia was also significantly higher than the pre-ischemia value (7.4 \pm 0.7 vs. $2.5 \pm 0.3(\mathrm{P}=0.001)$ and $5.8 \pm 0.4$ vs. $2.2 \pm 0.2$ $(\mathrm{P}=0.001)$, respectively (Figure 4$)$.

Administration of curcumin in the $\mathrm{C}-30$ and $\mathrm{C}-45$ groups attenuated the ischemia-induced rise in TNF- $\alpha$ compared to the V-30 and V-45 group, respectively ( $\mathrm{P}<0.05$ for both comparisons).

The pre-ischemia TNF- $\alpha$ in all the four groups were comparable ( $\mathrm{P}>0.05$ for all comparisons). However, the post-ischemia TNF- $\alpha$ in V-45 was higher than the one in $\mathrm{V}-30$ but did not reach statistical significance $(\mathrm{P}=0.07)$ and the level in $\mathrm{C}-45$ was significantly higher than the level in $\mathrm{C}-30(\mathrm{P}=0.01)$.

\section{Histological studies}

The right non-ischemic kidneys in all the groups had normal architecture (score 0, Figure 5A and Figure $5 \mathrm{~B}$, respectively). The left ischemic kidneys in the $\mathrm{V}-30$ group, showed acute tubular necrosis and tubular distension with necrotic material, involving approximately all tissue fields (score 4, Figure 5C) whereas the left kidney in the C-30 group showed these histological abnormalities in $50 \%$ of the fields and the rest of the fields showed normal histology (score 2, Figure 5D). The left kidneys in both V-45 and C-45 groups showed similar morphological features with tubular necrosis involving all fields (score 4, Figure 5E and Figure 5F, respectively).

\section{Discussion}

In the current study, we have demonstrated that the administration of curcumin for five days prior to warm renal IRI and for one day after the renal insult resulted in a significant attenuation in the associated rise in TNF- $\alpha$ and in the histological features at least in the 30-minute ischemia group but did not have a protective effect on the glomerular or tubular renal functional parameters when measured two days after the ischemia.

Limited number of studies has shown that curcumin has a protective effect on some markers of renal injury including histological features early after IRI (Shoskes 1998, Shahed et al. 2001, Bayrak et al. 2008). However, there is no specific data on the effect of curcumin on the renal functional parameters such as the GFR, RBF or tubular functional parameters following this insult. In one study, curcumin was found to significantly ameliorate the rise in the serum level of urea following IRI (Bayrak et al. 2008). However, it is wellknown that serum urea is an inaccurate measure of the GFR. Indeed, in the same study, curcumin did not attenuate the ischemia-induced rise in serum creatinine. The results of the current study were in agreement with these findings as there was no significant improvement in the GFR as measured by the clearance of inulin which is a more precise measure of GFR compared to serum creatinine (Stevens et al. 2006). 


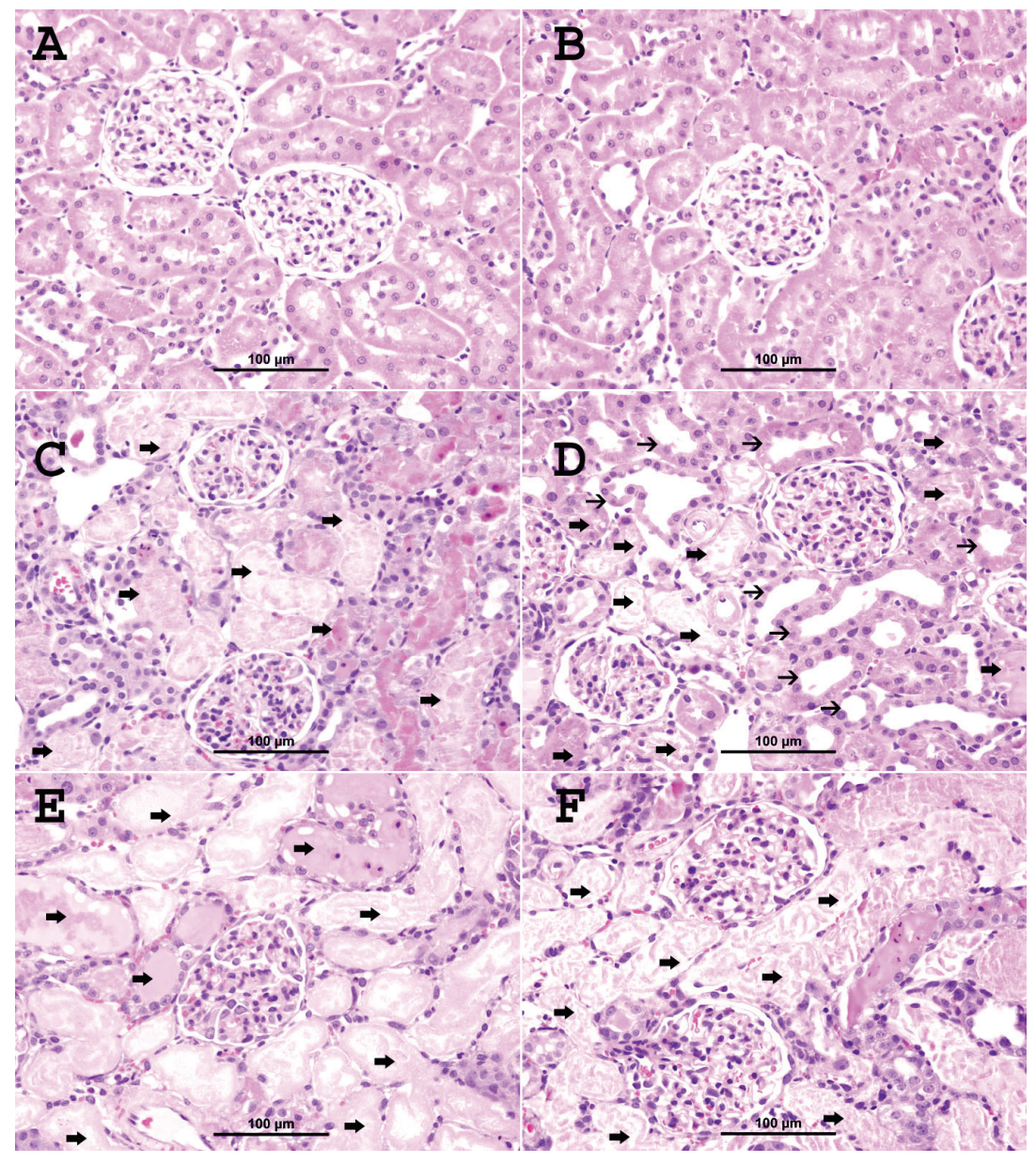

Fig. 5. Light microscopic features of the rats kidney sections (H\&E). A: right kidneys from V-30 and V-45 groups. B: right kidneys from $\mathrm{C}-30$ and $\mathrm{C}-45$ groups. Both $A$ and $B$ shows normal morphology. C: left kidneys from V-30 showing acute tubular necrosis (thick arrows) and tubular distension with necrotic material, involving nearly all tissue fields. D: left kidneys from C-30 showing similar changes in only $50 \%$ of the fields, and normal tubules (thin arrows) in the rest. E: left kidneys from V-45 group. F: left kidneys from C-45 group. Both $E$ and $F$ demonstrate acute tubular necrosis and tubular distension with necrotic material, involving all tissue fields (thick arrows).
The other study which addressed the renal effects of curcumin in IRI was the one by Shoskes (1998). Following 30 minutes of ischemia, the administration of curcumin attenuated the rise in the serum creatinine which is not as accurate as inulin clearance for the measurement of GFR (Stevens et al. 2006). Furthermore, in their study, simultaneous contralateral nephrectomy was performed at the time of the ischemic insult. Contralateral nephrectomy was shown to cause an alteration in the GFR of the remaining kidney. For instance, Chamberlain and Shirley (2007) have demonstrated that contralateral nephrectomy resulted in an immediate decrease in the total GFR which took at least 8 days to reach a maximum of $77 \%$ of the total pre-nephrectomy level. Therefore, it is possible that the observed improvement in the serum creatinine with curcumin administration in the Shoskes study was due to its effect on the alterations in GFR which was associated with contralateral nephrectomy rather than those due to IRI. This is supported by the data from the current study and the findings of Ghosh et al. (2009) who demonstrated that curcumin significantly attenuated the rise in serum creatinine in 5/6 nephrectomized rats.
The results of this study appears to indicate that curcumin has some protective renal effects as indicated by its action on one of the acute inflammatory mediators in IRI (TNF- $\alpha$ in this case) (Meldrum et al. 2002, Kelly 2003) and by the improvement in the morphological features especially in the 30-minute ischemia group which received curcumin. The exact reason for the lack of simultaneous effect on the glomerular functional parameters is difficult to explain from the current results but may be due to the fact that the effect of curcumin was not substantial enough to be reflected as functional improvements. Alternatively, the curcumin might not have a significant effect on the main mediators involved in the hemodynamic alterations observed early following IRI such as nitric oxide, endothelins and angiotensin II (Lieberthal et al. 1989, Shibouta et al. 1990, Weight et al. 1996, Kontogiannis and Burns 1998). Instead, curcumin might have affected some of the inflammatory mediators which in turn affected the post-ischemia inflammatory process and renal morphological features but did not have a significant effect on the dynamics of the glomerular filtration and absorption process. Certainly, further research is required to address this issue and the long- 
term effect of curcumin on the renal function following IRI.

The lack of significant protective renal effect observed in this study is unlikely to be due poor bioavailability of curcumin as the same dose was shown to result in protective effects in various pathological conditions such as the IRI (Bayrak et al. 2008) and renal failure (Ghosh et al. 2009). Moreover, in the current study, curcumin administration caused a significant decrease in the post-ischemia serum levels of TNF- $\alpha$ and improved the ischemia-induced histological alterations indicating a good absorption and bioavailability of curcumin.

The lack of difference between the left renal artery blood flow immediately after reperfusion between the curcumin and vehicle groups was in agreement with the renal blood flow which was measured by the clearance techniques two days after reperfusion and indicates that curcumin did not affect the renal hemodynamics both immediately or two days after the ischemic insult. The drop in the renal blood flow immediately after the release of renal artery clamps compared to the pre-ischemic level were similar to other studies (Cristol et al. 1993, Hammad et al. 2000) verifying the validity of the model used in this study. This validity was also demonstrated by the significant drop in the GFR and RBF in the ischemic kidney compared to the normal contralateral kidney in all groups similar to the findings of other studies (Rubinstein et al. 2009).

In the current study, two periods of ischemia were used to investigate the effect of curcumin as the 30-min and 45-min ischemia represented the milder and more severe degrees of ischemia-induced renal injury, respectively. This was demonstrated by the less severe degree of alteration in the renal functional parameters such as the GFR and RBF and tubular parameters such as the fractional sodium excretion in the $30-\mathrm{min}$ ischemia group compared to the 45-min ischemia group. In addition, the rise in serum level of TNF- $\alpha$ and the morphological alterations were milder in the former.
These results were in agreement with the findings of other researchers (Axelsen and Cartwright 1979, Brady et al. 1996, Rippe et al. 2006). By using the two periods of ischemia, we were able to demonstrate that curcumin did not affect the alterations in the glomerular or tubular functions in any of the ischemic periods but did result in a significant attenuation in the rise in the serum levels of TNF- $\alpha$ in both periods. The effect of curcumin on the histological changes behaved somewhat differently as there was an improvement in the morphological features following 30 minutes of ischemia whereas there was a lack of similar histological improvements following 45 minutes of ischemia. This might be due to the fact that the injury in the 45-min group was so severe to be affected by the curcumin indicating that curcumin might have a mild protective effect in milder forms of renal injury. Whether curcumin would have significantly affected the renal functional parameters in a very mild form of ischemic renal injury such the 15 -min period is difficult to ascertain from the current study and further studies are required to clarify this point.

In conclusion, the administration of curcumin before and after IRI appears to have no significant protective effect on the total renal artery blood flow immediately after reperfusion or on the glomerular and tubular renal functions as early as two days following IRI despite the amelioration in some of the markers of renal injury and morphological features following shorter periods of ischemia.

\section{Conflict of Interest}

There is no conflict of interest.

\section{Acknowledgements}

We would like to thank Dr M.A.H. Al-Sultan (Animal House, FMHS) for the excellent animal care and Mrs Noura S. Al Bloushi and Mrs Hamda M. AlRomaithi for their technical help. This project was supported by a grant from the Faculty of Medicine and Health Sciences, United Arab Emirates University.

\section{References}

AXELSEN RA, CARTWRIGHT VE: Renal function, cortical blood flow and morphometry in ischaemic acute renal failure in the rat. Pathology 11: 629-640, 1979.

BAYRAK O, UZ E, BAYRAK R, TURGUT F, ATMACA AF, SAHIN S, YILDIRIM ME, KAYA A, CIMENTEPE E, AKCAY A: Curcumin protects against ischemia/reperfusion injury in rat kidneys. World J Urol 26: 285-291, 2008. 
BRADY HB, BRENNER B, LIEBERTHAL W: Acute renal failure. In: The Kidney. BM Brenner (ed), W B Saunders Company, Philadelphia, 1996, pp 1201-1252.

BRATTON C, MARSHALL E: A new coupling component for Sulfanil-amide determination. J Biol Chem 128: $537-$ $550,1939$.

CHAMBERLAIN RM, SHIRLEY DG: Time course of the renal functional response to partial nephrectomy: measurements in conscious rats. Exp Physiol 92: 251-262, 2007.

CRISTOL JP, THIEMERMANN C, MITCHELL JA, WALDER C, VANE JR: Support of renal blood flow after ischaemic-reperfusion injury by endogenous formation of nitric oxide and of cyclo-oxygenase vasodilator metabolites. Br J Pharmacol 109: 188-194, 1993.

GEKLE M, SILBERNAGL S: Mechanism of ochratoxin A-induced reduction of glomerular filtration rate in rats. J Pharmacol Exp Ther 267: 316-321, 1993.

GHOSH SS, MASSEY HD, KRIEGE R, FAZELBHOY ZA, GHOSH S, SICA DA, FAKHRY I, GEHR TW: Curcumin ameliorates renal failure in 5/6 nephrectomized rats: role of inflammation. Am J Physiol 296: F1146-F1157, 2009.

HAMMAD FT, DAVIS G, ZHANG XY, WHEATLEY AM: Intra- and post-operative assessment of renal cortical perfusion by laser Doppler flowmetry in renal transplantation in the rat. Eur Surg Res 32: 284-288, 2000.

JORDAN WC, DREW CR: Curcumin - a natural herb with anti-HIV activity. $J$ Natl Med Assoc 88: 333, 1996.

KELLY KJ: Distant effects of experimental renal ischemia/reperfusion injury. J Am Soc Nephrol 14: 1549-1558, 2003.

KONTOGIANNIS J, BURNS KD: Role of AT1 angiotensin II receptors in renal ischemic injury. Am J Physiol 274: F79-F90, 1998.

LIEBERTHAL W, WOLF EF, RENNKE HG, VALERI CR, LEVINSKY NG: Renal ischemia and reperfusion impair endothelium-dependent vascular relaxation. Am J Physiol 256: F894-F900, 1989.

LOTEMPIO MM, VEENA MS, STEELE HL, RAMAMURTHY B, RAMALINGAM TS, COHEN AN, CHAKRABARTI R, SRIVATSAN ES, WANG MB: Curcumin suppresses growth of head and neck squamous cell carcinoma. Clin Cancer Res 11: 6994-7002, 2005.

MAHADY GB, PENDLAND SL, YUN G, LU ZZ: Turmeric (Curcuma longa) and curcumin inhibit the growth of Helicobacter pylori, a group 1 carcinogen. Anticancer Res 22: 4179-4181, 2002.

MCCORD JM: Oxygen-derived free radicals in postischemic tissue injury. $N$ Engl J Med 312: 159-163, 1985.

MELDRUM KK, MELDRUM DR, MENG X, AO L, HARKEN AH: TNF-alpha-dependent bilateral renal injury is induced by unilateral renal ischemia-reperfusion. Am J Physiol 282: H540-H546, 2002.

MOHAN IK, KHAN M, SHOBHA JC, NAIDU MU, PRAYAG A, KUPPSAMY P, KUTALA VK: Protection against cisplatin-induced nephrotoxicity by Spirulina in rats. Cancer Chemother Pharmacol 58: 802-808, 2006.

NAGABHUSHAN M, BHIDE SV: Curcumin as an inhibitor of cancer. J Am Coll Nutr 11: 192-198, 1992.

REILLY PM, SCHILLER HJ, BULKLEY GB: Pharmacologic approach to tissue injury mediated by free radicals and other reactive oxygen metabolites. Am J Surg 161: 488-503, 1991.

RIPPE C, RIPPE A, LARSSON A, ASGEIRSSON D, RIPPE B: Nature of glomerular capillary permeability changes following acute renal ischemia-reperfusion injury in rats. Am J Physiol 291: F1362-F1368, 2006.

RUBINSTEIN I, ABASSI Z, MILMAN F, OVCHARENKO E, COLEMAN R, WINAVER J, BETTER OS: Hyperbaric oxygen treatment improves GFR in rats with ischaemia/reperfusion renal injury: a possible role for the antioxidant/oxidant balance in the ischaemic kidney. Nephrol Dial Transplant 24: 428-436, 2009.

SALH B, ASSI K, TEMPLEMAN V, PARHAR K, OWEN D, GOMEZ-MUNOZ A, JACOBSON K: Curcumin attenuates DNB-induced murine colitis. Am J Physiol 285: G235-G243, 2003.

SHAHED AR, JONES E, SKOSKES D: Quercetin and curcumin up-regulate antioxidant gene expression in rat kidney after ureteral obstruction or ischemia/reperfusion injury. Transplant Proc 33: 2988, 2001.

SHARMA OP: Antioxidant activity of curcumin and related compounds. Biochem Pharmacol 25: 1811-1812, 1976.

SHIBOUTA Y, SUZUKI N, SHINO A, MATSUMOTO H, TERASHITA Z, KONDO K, NISHIKAWA K: Pathophysiological role of endothelin in acute renal failure. Life Sci 46: 1611-1618, 1990.

SHOSKES DA: Effect of bioflavonoids quercetin and curcumin on ischemic renal injury: a new class of renoprotective agents. Transplantation 66: 147-152, 1998. 
SRIMAL RC, DHAWAN BN: Pharmacology of diferuloyl methane (curcumin), a non-steroidal anti-inflammatory agent. J Pharm Pharmacol 25: 447-452, 1973.

STEVENS LA, CORESH J, GREENE T, LEVEY AS: Assessing kidney function--measured and estimated glomerular filtration rate. $N$ Engl J Med 354: 2473-2483, 2006.

SUGIYAMA Y, KAWAKISHI S, OSAWA T: Involvement of the beta-diketone moiety in the antioxidative mechanism of tetrahydrocurcumin. Biochem Pharmacol 52: 519-525, 1996.

WEIGHT SC, BELL PR, NICHOLSON ML: Renal ischaemia - reperfusion injury. Br J Surg 83: 162-170, 1996. 